\title{
Diseño, validez y confiabilidad del instrumento de observación "indicadores de pericia de la enfermera"
}

\author{
Design, validity and reliability of the instrument \\ for observation "indicators of experts of the nurse"
}

"Yariela González Ortega•

\section{Resumen}

Intoducción: El Propósito de este estudio fue diseñar y validar el instrumento de observación "indicadores de pericia de la enfermera", con el objetivo de identificar la expertes de la enfermera clínica, al proporcionar cuidado de relación interpersonal al paciente hospitalizado. Metodología: Esta investigación se realizó en varias fases: la primera consistió en el diseño y elaboración del instrumento. La segunda, comprendió el proceso de validez. La validez de contenido fue realizada a través de un panel de cuatro expertos y para la validez de criterio se utilizó la correlación de Pearson, el instrumento de indicadores de pericia se aplicó a una muestra de 220 enfermeras, con el instrumento de Cuidado de comportamiento profesional. La tercera fase fue de confiabilidad, se realizó tomando en cuenta a observadores entrenados, utilizando una muestra de diez enfermeras y aplicando la prueba de concordancia de Kappa de Cohen. Resultados: se obtuvo una validez de contenido por ítems entre 1 y 0,75 y una validez global de 0,90. En la confiabilidad se obtuvo un grado de concordancia entre los observadores de 90,9\%. El valor $r=0,662$. La regresión lineal dio la ecuación: $y=a+b$ x, en donde "b" representa la pendiente, cuyo valor fue de 0,65 y "a" la ordenada al origen, fue de 13,02.Conclusión: El instrumento, es práctico para clasificar a las enfermeras, en cuanto a su expertes de relación interpersonal; ya que, presentó propiedades psicométricas aceptables. El instrumento de observación, "indicadores de pericia", predice el cuidado de comportamiento profesional, a través de una relación de tipo lineal.

- Doctora en Enfermería

Docente de la Facultad de Enfermeríade la Universidad de Panamá

Correspondencia: yarielag11@gmail.com

RECIBIDO:11 DE DICIEMBRE DE 2009.

ENVIADO: 10 DE ENERO DE 2010

ACEPTADO:29 OCTUBRE DE 2010. 


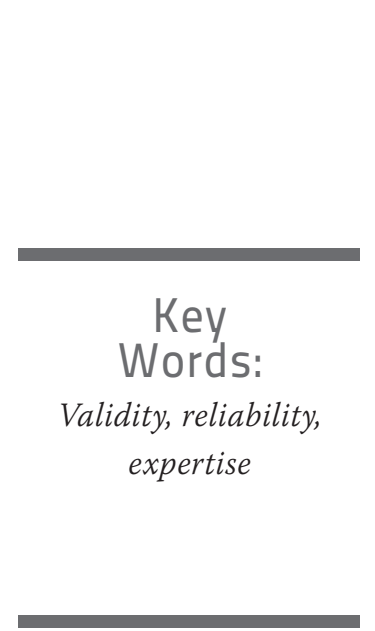

AbStRact

Introduction: The purpose of this study was to design and validate the instrument of observation "Indicators of Nurse Expertise, with the aim of identifying the expertise of the clinical nurse, providing care of interpersonal relationship to hospitalized patients., Methodology: This methodological research was done in several phases: the first involved in the design and development of the instrument, and the second, the process of validity. Content validity was conducted by a panel of four experts and the validity of criterion, a Pearson correlation between the indicators of expert instrument with a sample of 220 nurses was used, with the instrument of Professional Caring behavior. The third phase involved the reliability was performed taking into account trained observers, using a sample of ten nurses and a Cohen's Kappa agreement test. Results: We obtained a content validity of items between 1 and 0.75 and an overall validity of 0.90 . In the reliability, an agreement degree among observers of $90.9 \%$ was obtained, $r=0.662$. Linear regression with the equation: $y=a+b x$, where " $b$ " represents the slope, with a value of 0.65 and "a" the intercept, was 13.02. Conclusion: The instrument is convenient to classify nurses, in terms of interpersonal relationship expertise, as presented acceptable psychometric properties. The instrument of observation, "Indicators of Expertise," predicts professional caring behavior, through a linear relationship.

\section{INTRODUCCIÓN}

La validez y confiabilidad del instrumento de observación "indicadores de pericia de la enfermera", se realizó como parte del proyecto de investigación "Cuidado de relación interpersonal según experiencia práctica de la enfermera" con el objetivo de identificar la expertes de la enfermera, al proporcionar cuidado de relación interpersonal al paciente hospitalizado.

El tener este instrumento válido y confiable se convierte en un marco de referencia que contribuye a conocer y medir la pericia de la enfermera y contribuye a identificar a la disciplina de enfermería en su acto de cuidar.

Un aspecto de la práctica es la experiencia, cuyo significado etimológico deriva del latín expertus. Para el ámbito de enfermería esta experiencia juega un papel fundamental en el desarrollo de los rasgos de la práctica; dado que, se asocia a la acumulación del conocimiento y la habilidad, estrechamente vinculados con la experiencia. ${ }^{1}$

La práctica de enfermería, basada en el cuidado, es un campo de experiencia, ${ }^{2}$ en donde se integran los aspectos cognitivos, de comportamiento, de habilidades, valores y creencias que se aplican al cuidado del individuo, familia y comunidad. De tal manera, que esta práctica debe ser flexible, creativa, individualizada, con orientación social, compasiva y experta. ${ }^{3} \mathrm{El}$ cual debe ser aplicado en el campo clínico para tomar decisiones con base a un juicio clínico y a acciones reflexivas, que respondan a las necesidades particulares de cada persona. ${ }^{3,4,5}$

Del mismo modo, la práctica de enfermería exige también un nivel de dominio, que surge como resul- tado de un aprendizaje individual ${ }^{4}$ que se da a través de cinco fases, que van de forma gradualmente ascendentes de principiante a experto y que depende de la formación teórico - práctica, la experiencia, el interés, la pericia, la capacidad de las enfermeras y el contexto donde se realiza.

De manera que, a medida que la enfermera va adquiriendo experiencia clínica, también cambia su orientación intelectual, integra,y selecciona sus conocimientos; además, su habilidad de actuación se fundamenta en el contexto en que la enfermera es experimentada, así, un cambio de ambiente familiar o un contexto poco conocido significará que la enfermera podrá funcionar como un experto. ${ }^{4,6}$

La experiencia práctica, conocida como la acumulación progresiva de la habilidad y el conocimiento de 
la enfermera, se identifica, a través de atributos que presentan las enfermeras expertas como: seguridad en su práctica y en proporcionar cuidados, conocimiento de lo que realiza, receptividad y motivación hacia a otros, e interés por comprenderlo, relacionarse con ellos y tratarlos con dignidad. ${ }^{1,7}$

Actuar como una enfermera experta, implica conocer y percibir sus carencias con habilidad y destreza, ${ }^{1}$ poseer capacidad de infundir fe y esperanza para que los pacientes afronten sus problemas de salud, significa estar atenta al paciente para escucharlo y responder a sus dudas en el momento oportuno, ${ }^{8}$ mantener la serenidad y la madurez en situaciones difíciles y estresantes, sin que sea dañina para ella o para el paciente.

Además de todos estos atributos mencionados anteriormente, la enfermera debe conocerse a sí misma, para que pueda entrar en el campo de los significados de la otra persona, ${ }^{8}$ y fomentar la manifestación de las emociones y pensamientos del paciente.

Las investigaciones han demostrado distintos enfoques sobre los aspectos instintivos y de competencias de la enfermera experta. Por ejemplo, dominios y condiciones surgidas de las enfermeras expertas sobre su propia práctica clínica, ${ }^{8}$ sobre la habilidad del cuidado de enfermería según diferentes niveles de destreza clasificadas en: iniciante, patrón y veterano. ${ }^{9} \mathrm{Se}$ ha determinado la clasificación de la experiencia en cuatro tipos: tecnológica, tradicionalista ,especialistas, humanísticas existencialista. ${ }^{10} \mathrm{Y}$ se han diseñado y validado instrumentos para medir la pericia o expertes. ${ }^{11}$

\section{Metodología}

Esta investigación se desarrolló durante los meses de mayo - julio de 2007. Este tipo de estudio aborda el desarrollo, validación y evaluación de instrumentos, siendo su objetivo principal el desarrollar herramientas confiables, eficaces y funcionales que se puedan utilizar en otras investigaciones. ${ }^{12}$ Además de permitir aplicar pruebas psicométricas para establecer la validez y confiabilidad del instrumento de observación "indicadores de pericia de la enfermera".

El estudio se realizó en cuatro fases: diseño del instrumento, validez de contenido, validez concurrente y confiabilidad.

Fase 1: La primera fase consistió en diseñar el instrumento de indicadores de pericia, su elaboración se basó en una búsqueda bibliográfica extensa de implicaciones teóricas de expertes y en resultados de investigaciones referentes a las habilidades y características que los versados han identificado como las habilidades de una enfermera experta, lo que permitió describir cada comportamiento de experta(o) a observar.

De todo este marco presentado, resultó una primera versión del instrumento de observación con 20 ítems, distribuidos en tres dimensiones: dimensión de aspectos cognitivos que contiene siete ítems con indicadores de toma de decisiones, seguridad y confianza al actuar; dimensión de aspectos de comportamiento con siete ítems, que agrupa indicadores de actitudes dentro de una relación con el paciente y la dimensión de comunicación con seis ítems, que agrupa aspectos de técnicas en la comunicación efectiva.
Cada unidad de análisis se midió, a través de una escala tipo Likert que va de 1 a 4, donde 1 representa la puntuación más baja y 4 la puntuación más alta, cuyas categorías son llamadas nunca, casi nunca, casi siempre y siempre. Se utilizó este tipo de escala, ya que, está permite utilizar la correlación con otro instrumento (patrón estándar).

Fase 2: La segunda fase consistió en la validez de contenido, se utilizó un panel de cuatro enfermeras expertas que respondían a los siguientes criterios de inclusión: ser enfermeras, y tener como mínimo dos años de experiencia docente. Se entregó a cada experto, el instrumento de concordancia de expertos cuya estructura estaba diseñada con cinco columnas, la primera contenía todos los indicadores del instrumento diseñado en este estudio (indicadores de pericia); y las otras cuatro columnas corresponden a las categorías de una escala Likert llamadas muy pertinente, pertinente, casi pertinente y no pertinente.

La razón de validez de contenido (RVC), se obtuvo a través de los datos obtenidos en el instrumento de concordancia, realizado por los expertos. Para obtener este valor, se utilizó las ecuaciones [1] y [2], ${ }^{13}$ las cuales son una modificación del modelo de Lawshe, sobre el cálculo de la validez de contenido de un instrumento.

a) Ecuación para el cálculo de validez de contenido para cada ítem.

$$
C V R^{\prime}=\frac{n_{e}}{N} \quad[1]
$$

Donde:

$\mathrm{n}_{\mathrm{e}}=$ número de panelistas que tienen acuerdo en la categoría "esencial" 
$\mathrm{N}=$ número total de panelistas

b) Ecuación para el cálculo de validez de contenido global del instrumento.

$$
C V I=\frac{\sum_{i=1}^{M} C V R i}{M}
$$

Donde:

CVRi = Razón de Validez de Contenido de los ítems aceptables de acuerdo con el criterio de Lawshe modificado (Lawshe, C. H. En: Tristán A. 2007).

$\mathrm{M}=$ Total de ítems aceptables de la prueba.

La interpretación de los resultados obtenidos mediante la aplicación de la ecuación [1] y [2], se compararon con los criterios establecidos por Lawshe, ${ }^{14}$ donde plantea que si se tiene más del $50 \%$ de acuerdos entre jueces, se debe considerar que el ítem tiene un cierto grado de validez de contenido.

Fase 3: la tercera etapa consistió en medir la validez de criterio concurrente,para ello, se utilizó una muestra de 400 pacientes a los que se aplicó el instrumento Estándar o Patrón de Oro (Cuidado de comportamiento profesional) y 220 enfermeras a los que se le aplicó el instrumento de observación indicadores de pericia.

El instrumento de Cuidado de Comportamiento Profesional (forma A), ${ }^{16}$ elaborado con el objetivo de valorar la percepción del cuidado de comportamiento de las enfermeras en pacientes adultos hospitalizados. El instrumento utiliza una escala tipo Likert con cuatro categorías, con un rango de 4 - 1 puntos, que va de un grado de total acuerdo a un total desacuerdo, y cuenta con un total de 28 ítems. Presenta cuatro factores: no comprometido", (varianza explicada de 33,6\% y 10 ítems negativos);interacción con la familia - paciente", (varianza explicada de $5,7 \%$ y siete ítems); cortesía (varianza explicada de $5 \%$ y cinco ítems) y relación (varianza explicada de $4,1 \%$ y seis ítems). ${ }^{17} \mathrm{La}$ validez de contenido es de 0,90 , con una validez de constructo, que presenta cargas factoriales por ítems entre 0,46 y 0,68. La consistencia interna, a través del coeficiente de Alfa de Cronbach, es de 0,92 y la estabilidad a través de test - retest reportó un valor de 0,81 , con un $\alpha 0,05 .^{17}$

El procedimiento para realizar la validez de criterio concurrente y predictiva fue la aplicación del instrumento de Cuidado de Comportamiento Profesional a los pacientes hospitalizados para obtener su percepción con respecto al comportamiento de la enfermera al proporcionarle cuidado de enfermería, simultáneamente se aplicó el instrumento de observación a la enfermera por los observadores. Los datos obtenidos, fueron procesados con el programa estadístico SPSS versión 11.5 y se les aplicó la prueba estadística de Correlación de Pearson, y la prueba de regresión lineal a las puntuaciones obtenidas en ambos instrumentos.

La confiabilidad de las puntuaciones asignadas por dos observadores se determinó utilizando el Coeficiente de Kappa de Cohen.
Fase 4: la cuarta y última etapa, consistió en realizar la confiabilidad, para ello, se seleccionó una muestra de diez enfermeras que respondían a los siguientes criterios de inclusión: ser enfermeras que laboran de planta en los servicios de hospitalización de medicina y cirugía, que prestarán atención directa al paciente, y que laboran en los tres turnos.

El procedimiento para efectuar la confiabilidad, consistió en utilizar dos observadores, siendo uno de ellos el propio investigador y el segundo observador fue capacitado con respecto a las variables y categorías del instrumento de observación, y sobre las diferentes formas de cómo podría manifestarse en la práctica cada variable que se deseaba medir.

Cada enfermera de la muestra, fue observada simultáneamente, por el investigador y personal capacitado, en el momento en que se encontraba en contacto directo con el paciente o realizando un procedimiento por un período de tiempo de 60 minutos.

Ambas en el período de la mañana y la tarde. Una vez realizado esto se procedió a completar el instrumento de observación "indicadores de pericia". Posteriormente se entregó el instrumento a la enfermera para que fuera completado por ella, con el objetivo de disminuir el sesgo de observación, esta última información no fue utilizada en este estudio.

La confiabilidad de las puntuaciones asignadas por dos observadores se determinó utilizando el Coeficiente de Kappa de Cohen. Este método identifica la concordancia de las apreciaciones que hacen dos jueces sobre el mismo atributo; de tal modo que, permite 
cuantificar el grado de concordancia más allá del azar.

Partiendo de este planteamiento, si los jueces hacen la clasificación de los sujetos de una manera similar, los valores de la tabla de contingencias mostrarán altas frecuencias en las celdas de la diagonal principal que, dudosamente, pueden deberse al azar y Kappa brinda valores cercanos a 1; de lo contrario, si los jueces clasifican a los sujetos con criterios distintos, Kappa tendería a 0, indicando que la mayor parte de la consistencia observada es atribuible al azar.

Los resultados se compararon con los rangos de interpretación de Landis J. y Koch que expresan los valores de Kappa asociados con el grado de concordancia (figura1).

Como principios éticos tomado en consideración en esta investigación fueron: la orientación de las enfermeras y los pacientes sobre el propósito del estudio, los beneficios, el derecho a la confidencialidad, al anonimato y a abandonar el estudio al momento de la aplicación de los instrumentos.

\section{Fig. 1. Interpretación de los valores de Kappa}

\begin{tabular}{r|l}
\hline $\begin{array}{l}\text { Valor } \\
\text { de Kappa }\end{array}$ & $\begin{array}{l}\text { Grado } \\
\text { de } \\
\text { concordancia }\end{array}$ \\
\hline$<0$ & Malo \\
0.0 .20 & Bajo \\
$0.41-0.40$ & Aceptable \\
$0.61-0.80$ & $\begin{array}{l}\text { Moderado } \\
\text { Substancial } \\
\text { (Buena) }\end{array}$ \\
0.81 y más & $\begin{array}{c}\text { Casi perfecto } \\
\text { (Excelente) }\end{array}$ \\
\hline & $\quad$ \\
\hline
\end{tabular}

\section{Resultados}

a) Validez de contenido

\section{Gráfica 1. Distribución de la puntuación obtenida en el instrumento de concordancia de expertos}

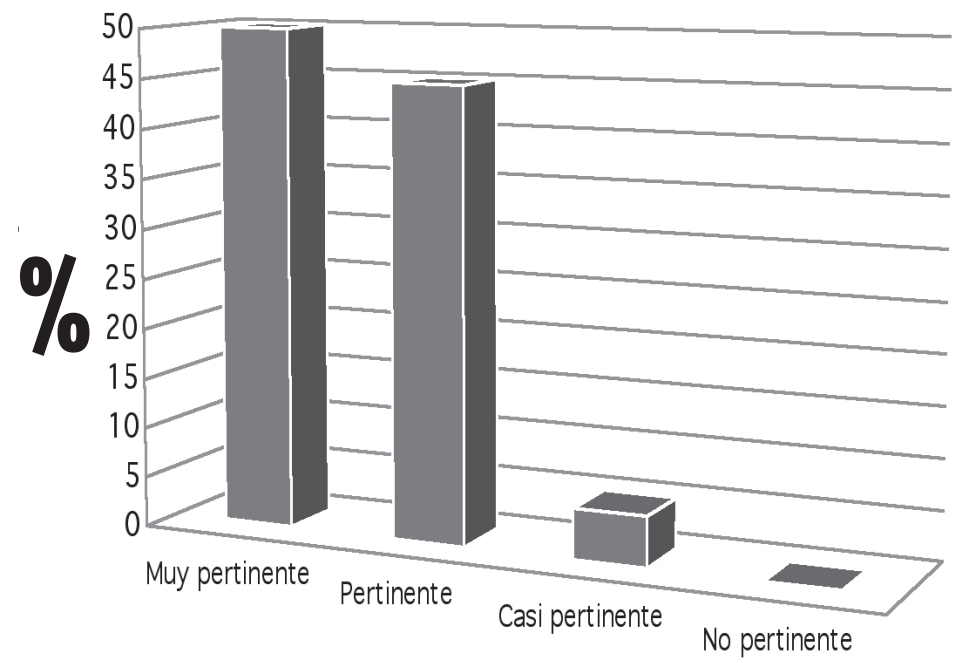

Fuente: Instrumento de concordancia de expertos.
La gráfica 1, muestra que el 95\% de los ítems del instrumento de observación "indicadores de pericia" fueron evaluados entre muy pertinentes y pertinentes, lo que quiere decir, que los ítems incluidos en el mismo, en su mayoría representan adecuadamente el concepto de expertes que se pretendía medir. Además, dentro de esta evaluación surgió la recomendación, por parte de los expertos, de mejorar la redacción de dos ítems.

Los resultados de la razón de validez de contenido por ítem presentó valores que oscilaban entre 0,75 a 1,00 en 19 de los ítems del instrumento, mientras que el ítems No 6 "ofrece cuidado de manera relajada" obtuvo una RVC de 0,33, valor inferior a lo establecido por Lawshe ; por lo que se tomó la de-

\section{Gráfica 2. Índice de validez de contenido por factores.}

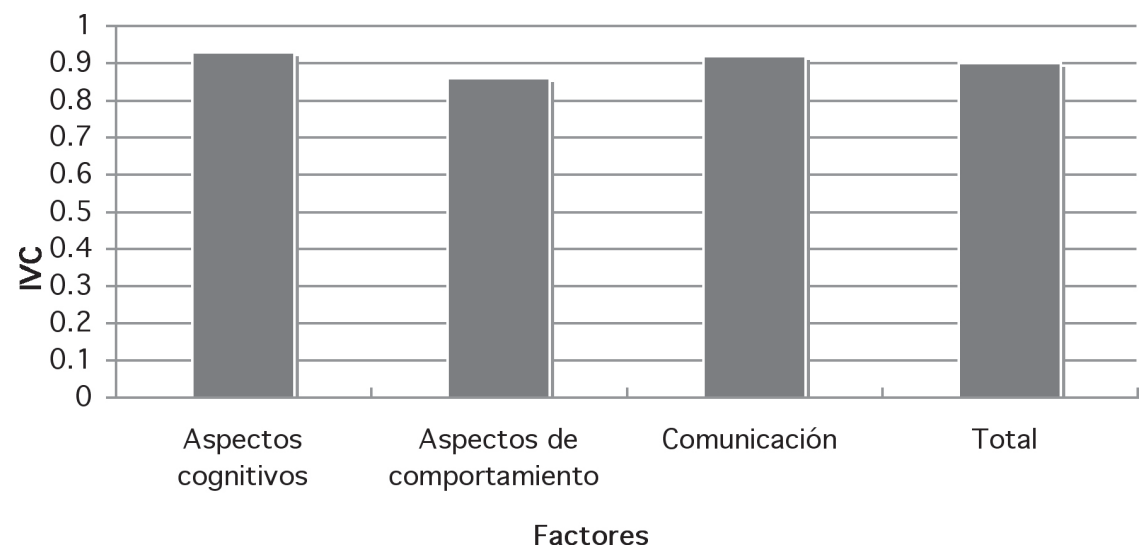

Fuente: Instrumento de concordancia de expertos. 
cisión de eliminarlo de la escala.

Por otra parte, el índice de validez de contenido correspondiente al instrumento global fue de 0,90 considerado como un valor alto, lo que demuestra que los ítems del mismo miden el constructo de manera coherente.

Los resultados de la RVC obtenidos por factores, oscilaron entre 0,86 y 0,93 , lo que indica que las unidades de análisis son claras, y que los indicadores representan adecuadamente los dominios o factores del instrumento.

Los expertos además, sugirieron agregar tres nuevos ítems dentro del factor de comunicación: el ítem No 20 "escucha de manera atenta al paciente", el ítem No 21 "conversa durante la intervención e ítem No 22 "utiliza tono de voz suave".

Al realizar las recomendaciones de los expertos, el instrumento en su versión final quedo con 22 ítems distribuidos en seis para los aspectos cognitivos, siete para aspectos de comportamiento y nueve ítems para los aspectos de comunicación.

b) Confiabilidad

\section{Tabla 1. Confiabilidad por ítem, a través del grado de concordancia de los observadores.}

\begin{tabular}{|c|c|c|c|}
\hline İtems & Kappa & İtems & Kappa \\
\hline 1 & 1,00 & 12 & 0,57 \\
\hline 2 & 0,62 & 13 & 0,00 \\
\hline 3 & 0,40 & 14 & 0,11 \\
\hline 4 & 0,74 & 15 & 0,44 \\
\hline 5 & 0,78 & 16 & 1,00 \\
\hline 6 & 1,00 & 17 & 0,69 \\
\hline 7 & 0,47 & 18 & 0,60 \\
\hline 8 & 0,21 & 19 & 0,70 \\
\hline 9 & 0,57 & 20 & 0,41 \\
\hline 10 & 0,82 & 21 & 0,62 \\
\hline 11 lente:Ins & $\begin{array}{r}0,85 \\
\text { nto de }\end{array}$ & ción aplica 22 & Is enfermeras \\
\hline
\end{tabular}

través de dos observadores.

La confiabilidad del instrumento, a través del Coeficien- te de Concordancia de Kappa, dio resultados altos en 17 ítems de 22 que contaba el instrumento y cuyo valores oscilaban entre 0,41 y más; hubo concordancia aceptable en tres ítems y concordancia baja en dos ítems, lo que puede interpretarse que hubo acuerdo entre los observadores en la gran mayoría de los ítems, reflejando que las observaciones no fueron al azar y que fueron manifestadas claramente.

\section{Gráfica 3. Distribución del grado de concordancia de los observadores que diligenciaron el instrumento de observación "indicadores de pericia".}

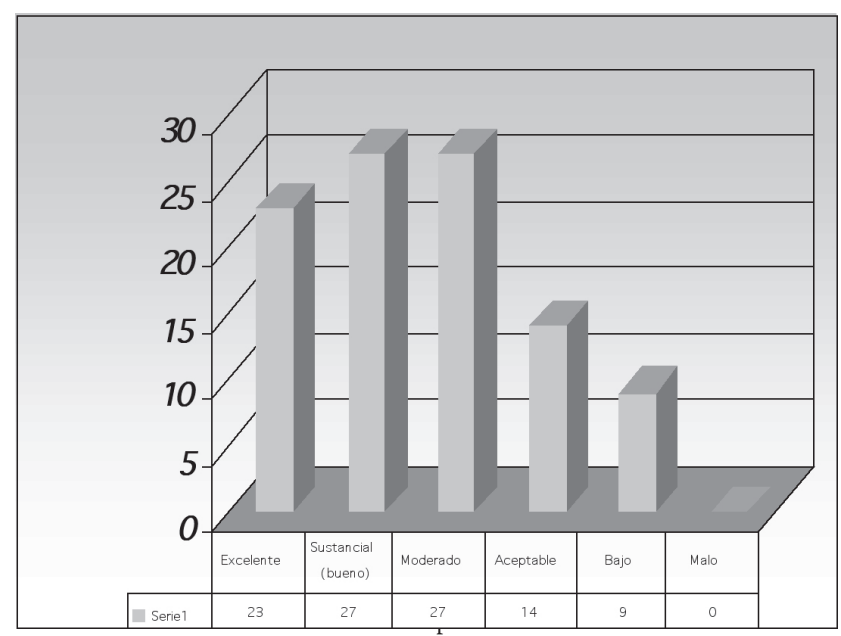

de dos observadores.

Siguiendo la interpretación de Landis J. y Koch G.G, la gráfica 3 representa la distribución de los ítems según categorías, donde cinco (23\%) de ellos, fueron clasificados como excelente, otros seis (27\%) como aceptables, seis $(27 \%)$ moderado, tres (14\%) aceptables y dos (9\%) como bajos, estos últimos fueron el ítem 13 "identifica los aspectos positivos del paciente para mejorar su estado físico" y el ítem 14 "toca al paciente, apropiadamente”. Esto refleja que la respuesta de los observadores pudo deberse a cierto grado de azar, motivo por el cual, para disminuir las discrepancias, probablemente, debido a la interpretación que le dio cada observador, se procedió a modificar, sutilmente, la redacción de los mismos para la versión final del instrumento.

Estos resultados en forma general, indican que el instrumento presentó concordancia entre los dos observadores de $90,9 \%$, lo que muestra que hubo una comprensión de los ítems por parte de los dos evalua- 
dores; por lo tanto, el instrumento mide las competencias que se pretende medir en esta investigación.

Por otra parte,para determinar, si existe o no, correlación entre la evaluación que obtuvieron las enfermeras con el instrumento cuidado de comportamiento profesional, comparado con la evaluación que recibieron las mismas enfermeras con el instrumento de observación "indicadores de pericia”, se determinó el Coeficiente de Correlación de Pearson, encontrándose un valor de 0,662; el cual muestra un coeficiente bueno.

c) Validez concurrente

\section{Gráfica 4.Regresión lineal entre las puntuaciones obtenidas por las enfermeras en el instrumento de cuidado de comportamiento profesional y las puntuaciones obtenidas en el instrumento de observación "indicadores de pericia".}

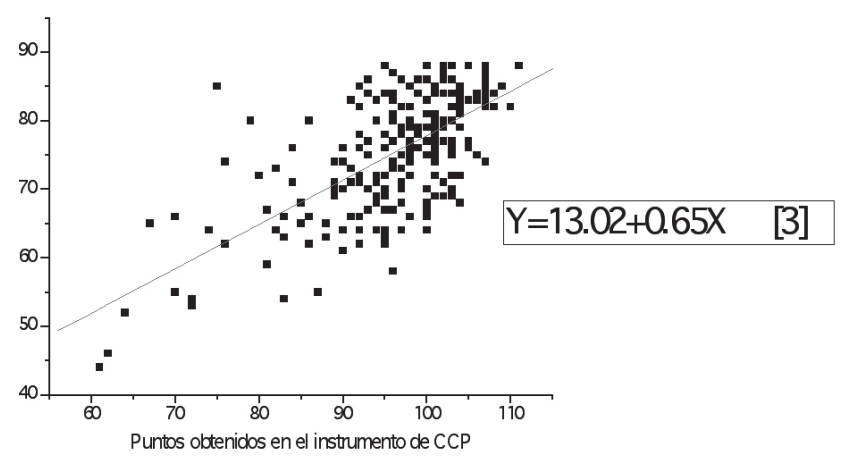

aplicado a las enfermeras e instrumento de cuidado de comportamiento profesional aplicado a los pacientes.

La Gráfica 4, muestra que existe una tendencia por una relación de tipo lineal; es decir, que mientras una variable aumenta, la otra también. La ecuación que relaciona estas variables, es de la forma: $y=a+b x$, en donde "b" representa la pendiente, cuyo valor fue de 0,65 y "a" la ordenada al origen, cuyo valor obtenido fue de 13,02.

Llevando estos resultados, a las variables del estudio, se encuentra que la relación entre las mismas está dada por la ecuación [3]:

Además, se repitió, esta prueba de validez concurrente, para dividir los instrumentos; de manera que se contara, solamente, con los ítems que se conside- raron comunes*, los cuales fueron diez. Después, se analizó la correlación entre ellos, y se obtuvo un mayor valor predictivo de $r=0,878$. Valor significativo con la prueba F de Fisher de 13,04 con significancia de 0,000 . Estos valores son mayores que la correlación obtenida previamente; por tanto, se concluye que el instrumento de indicadores de pericia tiene buena validez de criterio predictiva con el cuidado de comportamiento profesional.

\section{Discusión y Conclusiones}

El instrumento de observación, llamado indicadores de pericia, diseñado para clasificar la expertes de la enfermera, demostró una validez de contenido de 0,90 y una confiabilidad 90,9\% de concordancia (Coeficiente de Kappa). Lo que indica que presentó propiedades psicométricas aceptables, para ser utilizado como herramienta de evaluación, ampliando esto el campo de estudio dentro de la práctica de la enfermería.

$\mathrm{Al}$ analizar las correlaciones entre el instrumento de cuidado de comportamiento profesional, con el de indicadores de pericia, se encontró una buena correlación entre ambos, ya que las enfermeras que fueron bien evaluadas con el instrumento de cuidado de comportamiento profesional, también, fueron bien evaluadas con el instrumento de observación, "indicadores de pericia". Lo que indica, que ambos instrumentos se complemen$\tan y$ no se contradicen.

La prueba estadística de regresión lineal, aplicada a ambos instrumentos, demostró una relación de tipo lineal lo que indica que, si se tiene los puntos de cualquiera de los dos instrumentos, se puede obtener el del otro instrumento, mediante la ecuación encontrada en este trabajo; lo que implica, que a través de esta asociación se puede evaluar la expertes de la enfermera con el instrumento de observación "indicadores de pericia" o con el instrumento de cuidado de comportamiento profesional y viceversa permitiendo hacer la clasificación de la enfermera experta de una manera rápida y sencilla.

Al identificar la expertes en la enfermera se puede, a través de la gestión del cuidado, asignar una enfermera experta como tutora de una enfermera menos experta quien con sus conocimientos, madurez y habilidades sirva de guía y apoyo.

Se recomienda seguir desarrollando este instrumento a través de nuevas investigaciones, que permita clasificar la expertes de la enfermera con mayor precisión. 


\section{Referencias Bibliográficas}

1 Arbon P. Understanding Experience. Journal Of Clinical Nursing 2004; 13: $150-7$.

2 Kérouac S. El pensamiento enfermero. Barcelona: Masson; 1996.

3 Fawcett J. Analisis and evaluation of Contemporary nursing knowledge: analysis and evaluation of nursing models and theories. 2nd edition. U.S.A: Copyright by Davis Company; 2005.

4 Benner P. Práctica progresiva en enfermería. Manual de comportamiento profesional. España: $\mathrm{Hu}-$ rope S.A., traducido por R.A.A. 1987.

5 MarrinerTomey A. Modelos y teorías en enfermería. 5ta edición, España: Mosby/ Doyma, 2003.

6 Fisher AA, Fonteyn, ME. The nature of nursing expertise. In: Grobe, S.J. and Pluyter-Wenting, E.S.P., Editors, 1994. Nursing Informatics: An International Overview of Nursing in a Technological Era, Amsterdam, Elsevier Science, 1994 p. 331-335.
7 Bonner A. Recognition of expertise: an important concept in the Acquisition of nephrology nursing expertise Nursing and health sciences 2003; 5:123 -31.

8 Buller S, Butterworth T. Un estudio cualitativo de los elementos de enfermería International Journal of NursingStudies 200138 : 405 - 17.

9 Da Silva, AlcioneLeite. Habilidade intuitiva no cuidado de enfermagen. Revista Latino - Americana Enfermagen. 2003; 11(4): 429 - 35

10 Conway J E. Evolution of the species "expert nurse". an examination of the practical knowledge held by expert nurses. Journal of Clinical Nursing 1998; 7 (1): 75 - 82.

11 Meretoja R, Isoaha H, Leino - Kilpi, H. Nurse competence scale: development and psychometric testing. Journal of Advanced Nursing 2004; 53 (5) : 347 -52.

12 Polit D Hungler B. Investigación científica en ciencias de la salud. 6a ed. México: Editorial McGraw - Hill Interamericana. S.A. de C.V; 2000.
13 Tristán A. Modificación al modelo de Lawshe para el dictamen de validez de contenido de un instrumento objetivo. Avances en medición. 2008; 6(1):37-48.

14 Lawshe CH. A Quantitative approach to content Validity Personnel Psychology1975; 28:563-75 .

15 Watson J. Assessing and measuring caring in nursing and health science. U.S.A.: Springer Publishing Company; 2002.

16 Horner S. Información sobre la validez y confiabilidad del instrumento de comportamiento de cuidado profesional. Datos no publicados

17 Tristán A. y Vidal R. Manual de fórmulas de correlación. México: Trafford; 2006. 